\title{
FLOW VISUALISATION AROUND A SOLID SPHERE ON A ROUGH BED UNDER REGULAR WAVES
}

\author{
H.P.V.Vithana ${ }^{1}$, Richard Simons ${ }^{2}$ and Martin Hyde ${ }^{3}$
}

\begin{abstract}
Flow visualization using Volumetric Three-component Velocimetry (V3V) was carried out during laboratory tests to determine threshold shear stress and forces on idealized spherical stones. Incipient motion tests consisted of light weight spherical particles of specific gravity=1.19-2.65 and diameter=9.6mm-31.8mm. In-line and uplift forces on a $50 \mathrm{~mm}$ sphere sitting on a rough bed of similar spheres were measured using pressure transducers linked to tappings on the sphere surface. It was found that the Shields critical shear stress and stone protrusion has an exponential relationship. At an exposure of $0.2 \mathrm{~d}$ and less, bed protection is remarkably stable. Incipient motion data for currents are applicable for waves when the flow is fully developed.
\end{abstract}

Keywords: Shear stress; incipient motion; flow visualization

\section{INTRODUCTION}

This paper describes flow visualization tests carried out to understand the flow behavior near a spherical test stone sitting on a rough bed of same diameter spherical elements. The primary objective of the tests was to study the effect of stone protrusion on the Shields critical shear stress under the action of regular waves. The V3V measurements were used to obtain an insight into the flow structures that induce drag and uplift forces on a stone that cause stone displacement and give contribution to the Reynolds stresses to the flow region above the bed. The subsections under the discussion and results consist of (i) vortex shedding from a rough bed consisting of 50mm spheres, (ii) flow details around a $50 \mathrm{~mm}$ fully exposed sphere sitting on a bed of same diameter spheres, (iii) measured bed shear stress and wave friction factor, (iv) incipient motion data plotted against relative stone protrusion.

\section{EXPERIMENTAL SETUP}

\section{Apparatus}

The apparatus consisted of the $14.5 \mathrm{~m}$ long, $45 \mathrm{~cm}$ wide and $65 \mathrm{~cm}$ high UCL wave-current flume and a coplanar bed of $50 \mathrm{~mm}$ diameter billiard balls constructed on the flume bed (figure 3). The same diameter test stone was fixed above the coplanar bed over an interstice formed by three spheres (see figs. 1 and 2). $1.5 \mathrm{~mm}$ pressure tappings were provided on the test sphere surface on a meridian at 30 degree spacing which were then linked to the Honeywell 40PC001B2A type pressure sensors. The rough bed was $90 \mathrm{~cm}$ long spread across the width of the flume.

The apparatus for the measurement of incipient motion of stones was of the same dimensions except that it was constructed of $19 \mathrm{~mm}$ glass marbles. Spherical test stones, varying in diameter $(9.5-31.8 \mathrm{~mm})$ and density (specific gravity=1.19-2.65), were placed on top of the coplanar bed and were free to move.

\section{Volumetric Three-component Velocimetry}

Most complex flows of importance to scientists and engineers are three-dimensional (3D) in nature. The understanding and modeling of these flows require experimental tools that can provide instantaneous 3D flow information in a volumetric domain with good space and time resolution. Traditional PIV techniques only capture 2D slices of the flow fields and hence, cannot reveal the complete topology of 3D flow structures. Therefore, Volumetric Three-component Velocimetry (V3V) offers a novel technique for instantaneous volumetric measurement of the three-dimensional velocity fields.

\footnotetext{
1,2 Department of Civil, Environmental \& Geomatic Engineering, University College London, Gower Street, London WC1E 6BT, UK 
The core of $\mathrm{V} 3 \mathrm{~V}$ technique is a unique single-body, three-aperture, 12 mega-pixel camera for "point and shoot" 3D imaging. V3V uses volume illumination with $\mathrm{Nd}$ :YAG laser so that particles inside a large cubic volume up to $120 \times 120 \times 100 \mathrm{~mm}^{3}$ can be recorded based on two-frame double-exposure at 7.25 capture rate (see figures 4 and 5). Total of six images, two per each probe at time, $t=t$ (Frame A) and $t=t+\Delta t$ (Frame B) are captured. These are combined to locate the particle in volumetric 3D space using a triplet searching technique resulting in two 3D volumetric particle maps for Frame A and $\mathrm{B}$. Using 3D particle tracking technique, a single velocity vector corresponds to the motion of a single tracer particle to measure from a point in Frame A to a point in Frame B.

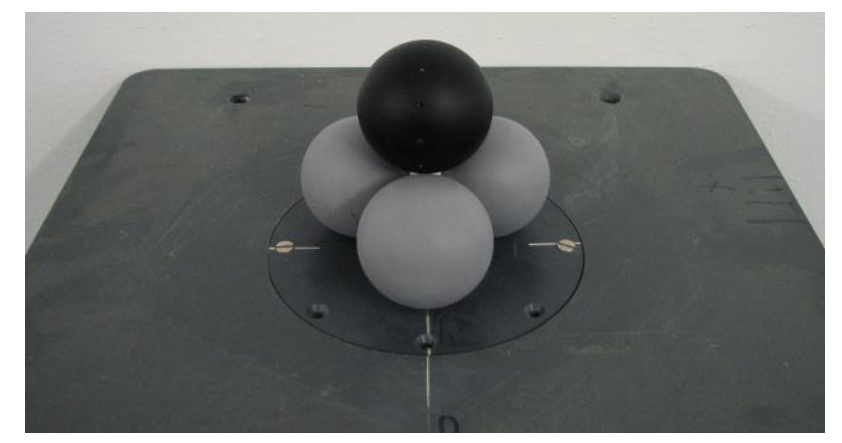

Figure 1. Sphere with pressure tappings (black).

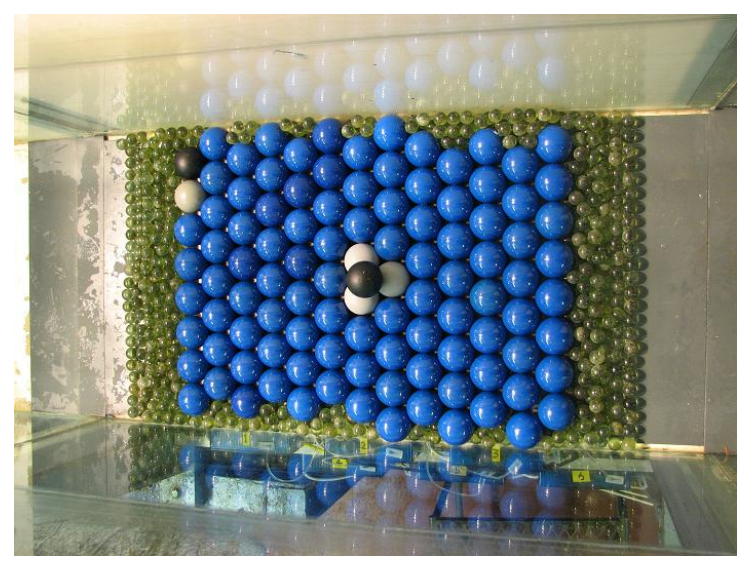

Figure 2. Fully exposed (over lying) test sphere on the coplanar bed.

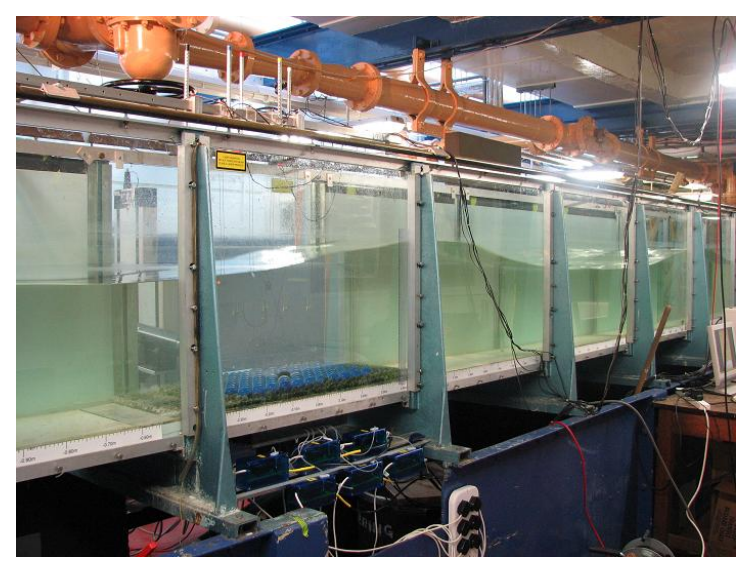

Figure 3. Waves running over the rough bed. Pressure sensors housed in boxes below. 


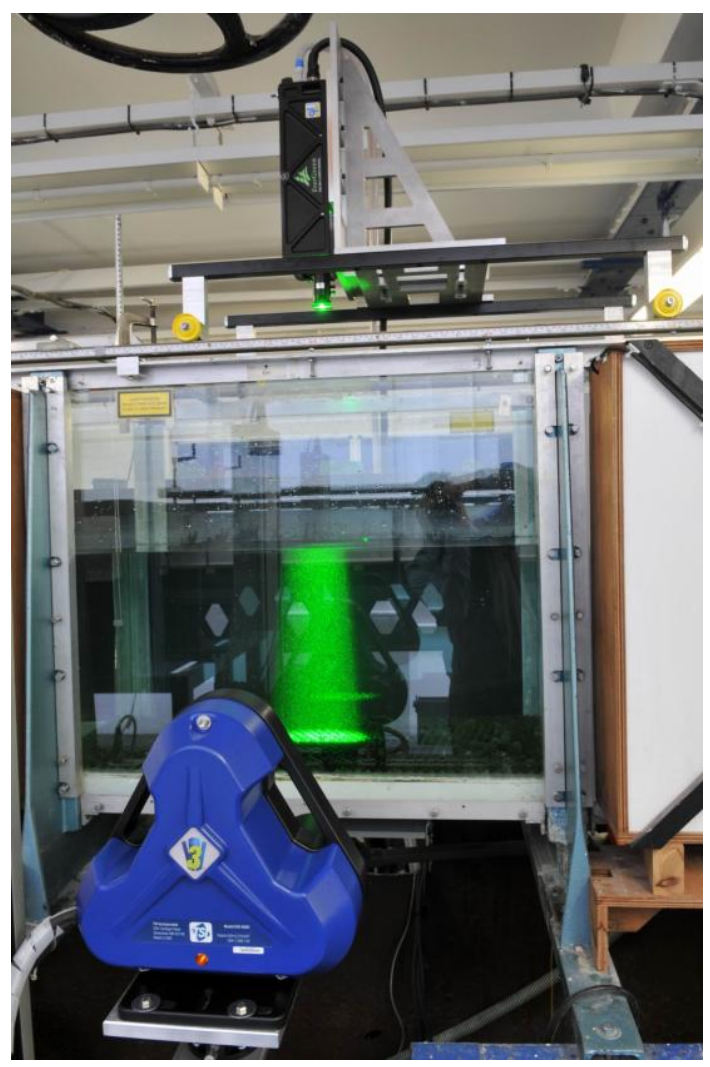

Figure 4. Camera probe and the laser beam.

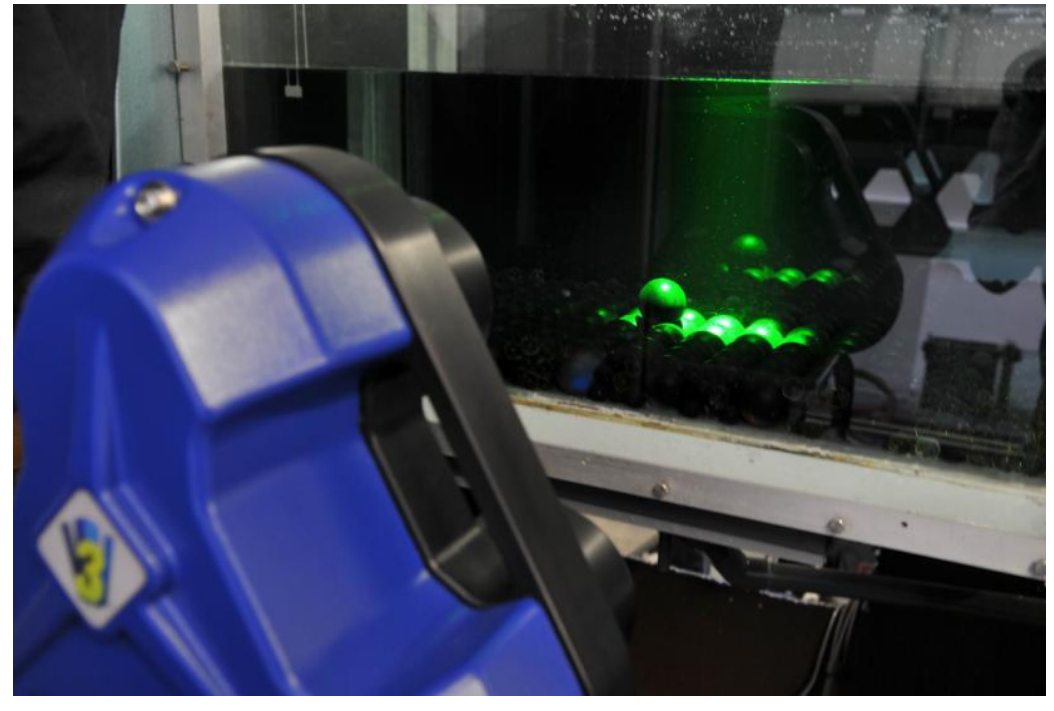

Figure 5. Test sphere illuminated by the laser beam. 


\section{DISCUSSION AND RESULTS}

\section{Vortex shedding from the roughness elements}

Vorticity isosurface of the flow structures near a rough bed of $50 \mathrm{~mm}$ spherical elements are shown in figures 7 to 13 . Figures 7 to 10 correspond to the vortex formation and figures 11 to 13 refer to the ejection and lifting off of coherent vortices into the main flow. Figure 6 shows the position of the V3V images in relation to the phase of the wave surface elevation. The near bed turbulence within a region approximately equal to diameter of the roughness elements, does not cease even when the average velocities fall to zero just before the flow reversal (see figure 11). This appears to agree with the Kajiura's (1968) assumption of constant eddy viscosity in a region $0.5 \mathrm{k}_{\mathrm{s}}$ above the bed. The flow Reynolds number was, $\mathrm{Re}=\mathrm{aU} \mathrm{m}_{\mathrm{m}} / \mathrm{v}=3.2 \times 10^{4}$.

\section{Flow around a $50 \mathrm{~mm}$ sphere}

Figures 15 to 17 show three consecutive V3V images describing the sequence of the formation and detachment of vortices from a 50mm sphere during the acceleration phase of the crest half cycle. The flow detachment occurs at the phase of the wave crest (figure 17) the process of which could impart an uplift force on the sphere. The measured wave height and period were $11.6 \mathrm{~cm}$ and 2 seconds. The Reynolds number, $\mathrm{Re}_{\mathrm{d}}=\mathrm{U}_{\mathrm{m}} \mathrm{d} / \mathrm{v}=11,300$ and $\mathrm{Re}=\mathrm{aU} \mathrm{U}_{\mathrm{m}} / \mathrm{v}=3.2 \times 10^{4}$. A raw image captured by the $\mathrm{V} 3 \mathrm{~V}$ camera probe in figure 14 shows the stone sitting on the coplanar bed.

\section{Shear stress and friction factor}

Shear stress and hence, wave friction factor were obtained by dividing net horizontal force on the pressure measuring sphere by its effective planar area. The pressure on the sphere was integrated assuming that the theoretical bed level is located $0.35 \mathrm{~d}$ below the crests of the roughness elements (see Sleath, 1987). It was found that time variation of the measured ensemble averaged shear stress agrees well with the cosine relationship with $\langle\tau\rangle=0.5 \mathrm{f}_{\mathrm{w}} \rho \mathrm{U}_{\mathrm{m}}{ }^{2}$ as the amplitude (see figure 18). The friction factor data $\left(\mathrm{f}_{\mathrm{w}}\right)$ also agreed well with the relationship of Simons et al. (2000), $\mathrm{f}_{\mathrm{w}}=0.33\left(\mathrm{a} / \mathrm{k}_{\mathrm{s}}{ }^{)-0.84}\right.$ where ' $\mathrm{a}$ ' is wave orbital amplitude and ' $\mathrm{k}_{\mathrm{s}}$ ' is Nikuradse roughess taken as $2.5 \mathrm{~d}$ (see figure 19).

\section{Incipient motion of stones}

In the tests first movement of a spherical stone sitting on a coplanar bed, was observed increasing wave heights in $0.5 \mathrm{~cm}$ increments until it starts rocking motion and then final displacement. The shear stress was calculated using $\tau=0.5 \mathrm{f}_{\mathrm{w}} \rho \mathrm{U}_{\mathrm{m}}{ }^{2}$ with $\mathrm{U}_{\mathrm{m}}$ obtained from linear wave theory (which agreed reasonably well with the ensemble averaged horizontal velocity measured using LDV). The tests were carried out for different stone protrusion levels. Figure 20 shows a plot of Shields critical shear stress versus relative stone protrusion, $\mathrm{p} / \mathrm{d}$. Critical shear stress increased exponentially when protrusion was decreased from $0.82 \mathrm{~d}$ (fully exposed condition). In addition to stone protrusion, it was found that the threshold stress also depends on wave period. When the wave period increased, the critical shear stress vs protrusion curve moved towards the curve of Chin and Chiew (1993) obtained for uni-directional currents. Flow visualization in figure 21 showed that lee-wake vortice downstream of a $19 \mathrm{~mm}$ stone was not established even at the peak velocity. Instead, a residual vorticity structure on top of the stone was present. This is because flow was not fully developed at the moment of inception of motion which occurred within the laminar $\left(\operatorname{Re}_{\mathrm{d}}<2000\right)$ or the transition flow regions for the majority of light weight particles. The rapid flow reversal due to small wave period inhibits the flow development. Therefore, under the field conditions where there is adequate time for the flow to achieve fully developed condition with long wave periods and high bottom velocities, incipient motion data obtained for currents should be applicable. 
Existence of significant uplift force is crucial for the displacement of stones. Figure 22 (Frame 15) shows the image just prior to the displacement of a light weight stone. The displacement occurred at Frame 16 (figure 23) when the vorticity structure has moved to the new position B close to the stone from position A inducing an uplift force on the stone. The time elapsed since the start of the wave run is 15.863 seconds (i.e. approximately 10 waves with $\mathrm{H}=8.8 \mathrm{~cm}, \mathrm{~T}=1.6$ ). This means, that the stone did not move even though 20 peaks in shear stress have passed. The stone displaced only when an eddy moved closer to the stone capable of producing an uplift. Therefore, the stone displacement depends on the joint probability of occurrence of both uplift and peak shear stress.

It was also empirically observed that a stone was remarkably stable when relative protrusion, $\mathrm{p} / \mathrm{d}$ was set to 0 (coplanar bed), 0.17 and 0.21 even for the largest bed shear stresses that could be produced in the wave flume. When the protrusion was increased to $0.24 \mathrm{~d}$, stones started to move again. Therefore, $0.2 \mathrm{~d}$ appears to be the threshold protrusion level below which there is no stone movement. The measurement of in-line and uplift forces showed that the force on a $50 \mathrm{~mm}$ stone with a protrusion of $0.216 \mathrm{~d}$ is very similar to that on a stone in a coplanar bed which is theoretically the most stable position (see figures 24 and 25).

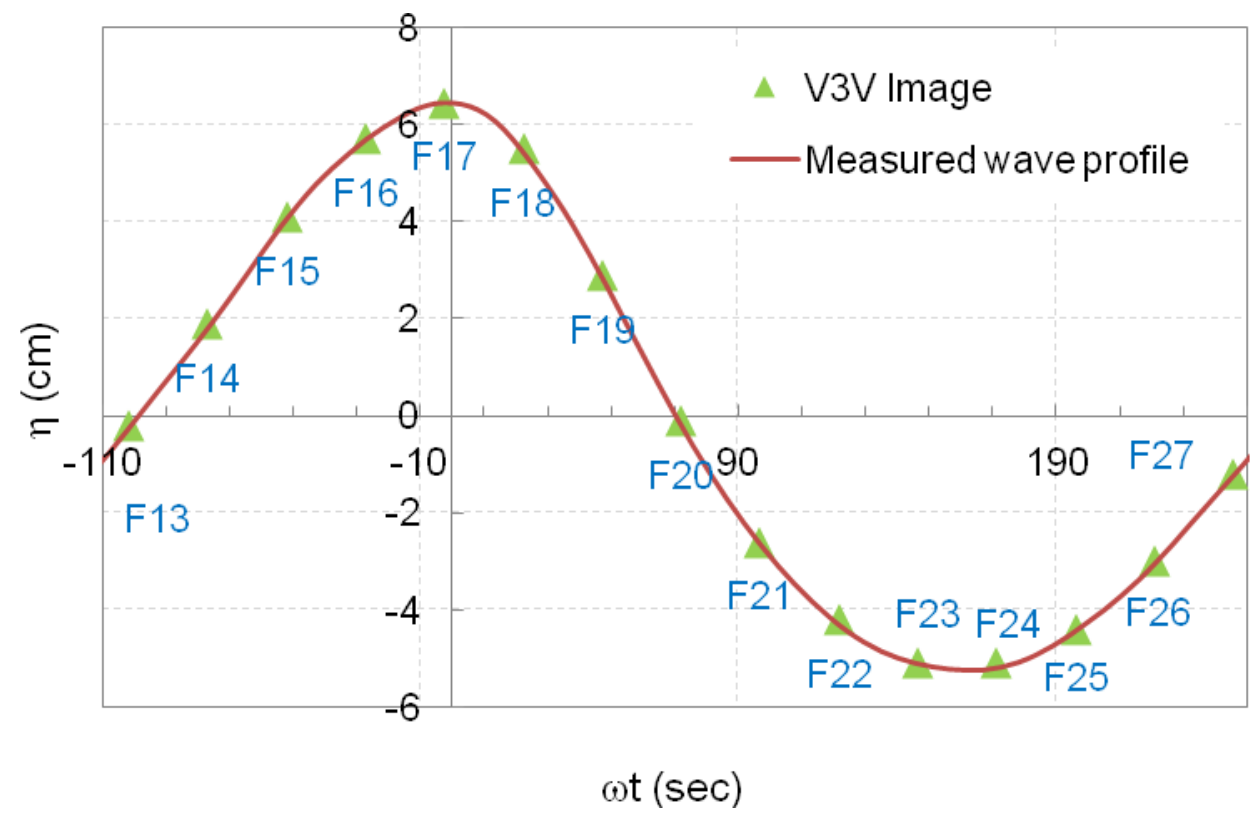

Figure 6. Measured wave surface profile. $H=11.63 \mathrm{~cm}, T=2 \mathrm{sec}$ with $\mathrm{V} 3 \mathrm{~V}$ image positions marked. 


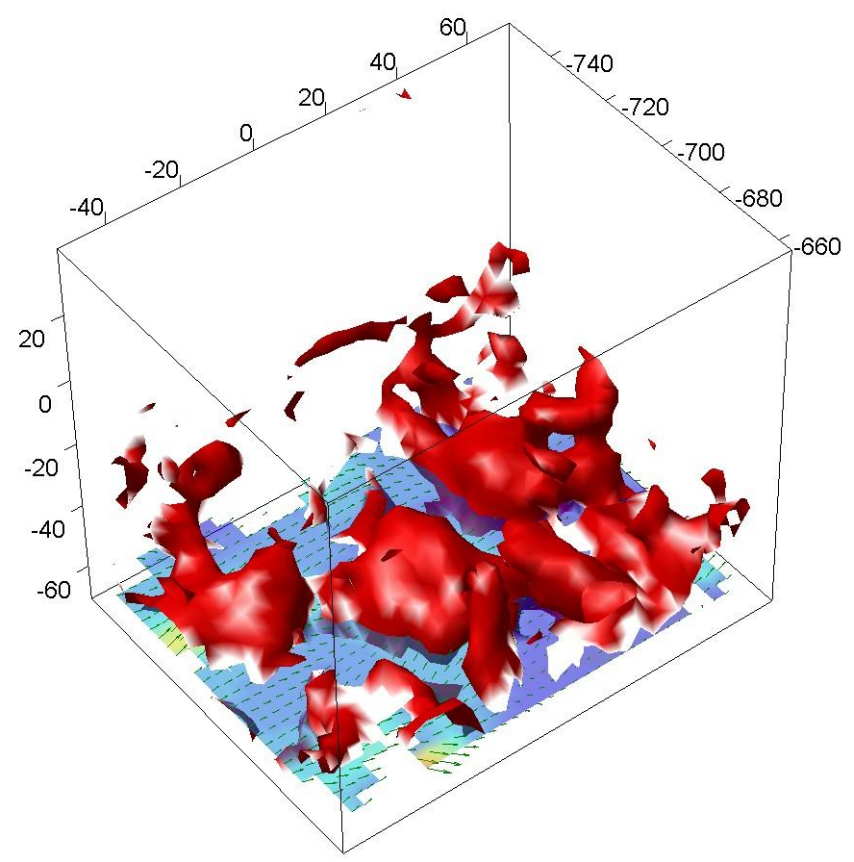

Figure 7. Vorticity isosurface, Frame 14.

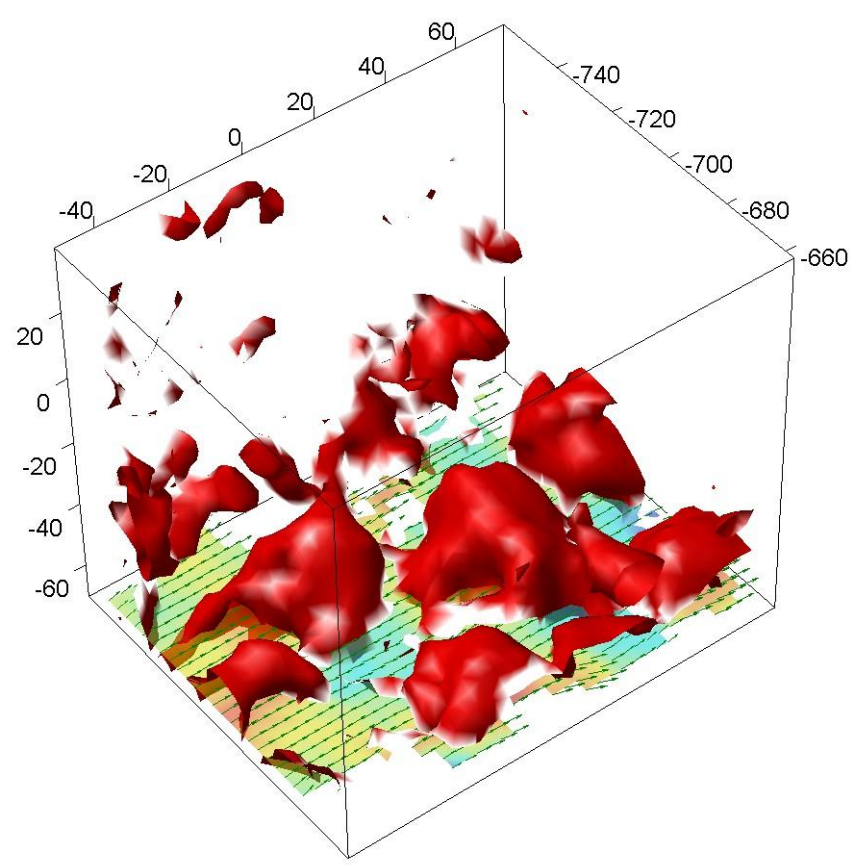

Figure 8. Vorticity isosurface, Frame 15. 


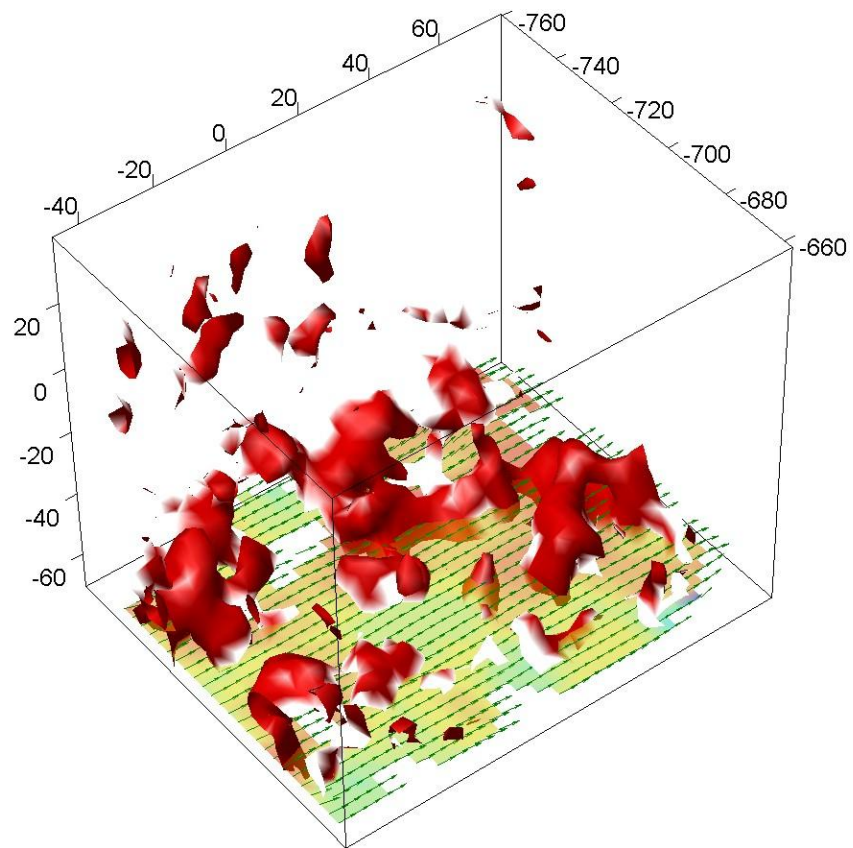

Figure 9. Vorticity isosurface, Frame 18.

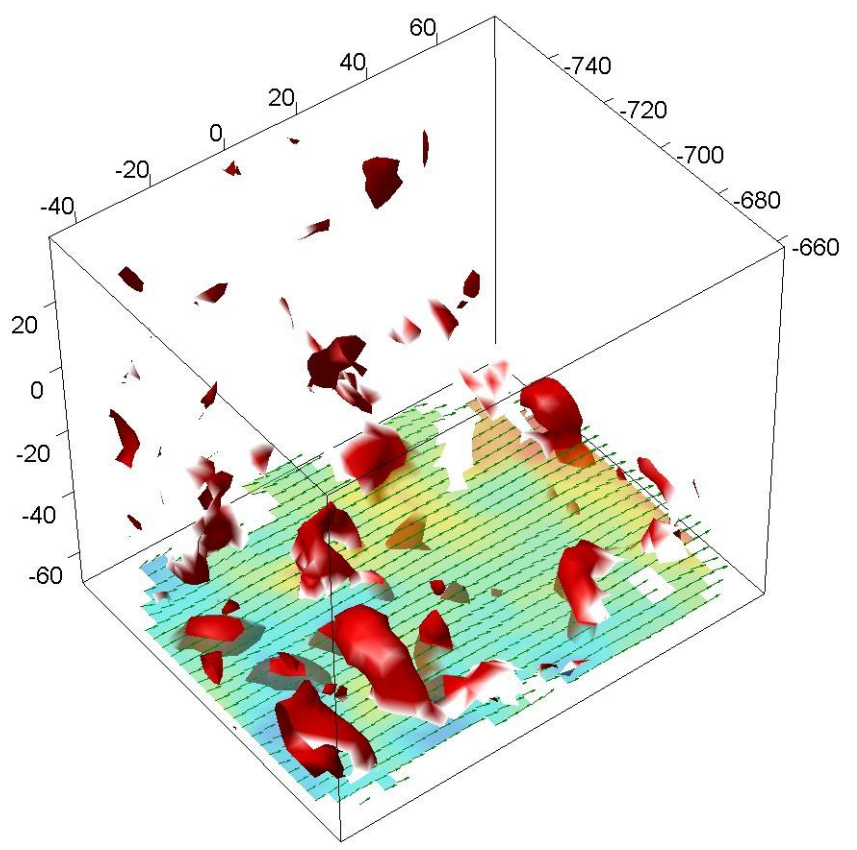

Figure 10. Vorticity isosurface, Frame 19. 


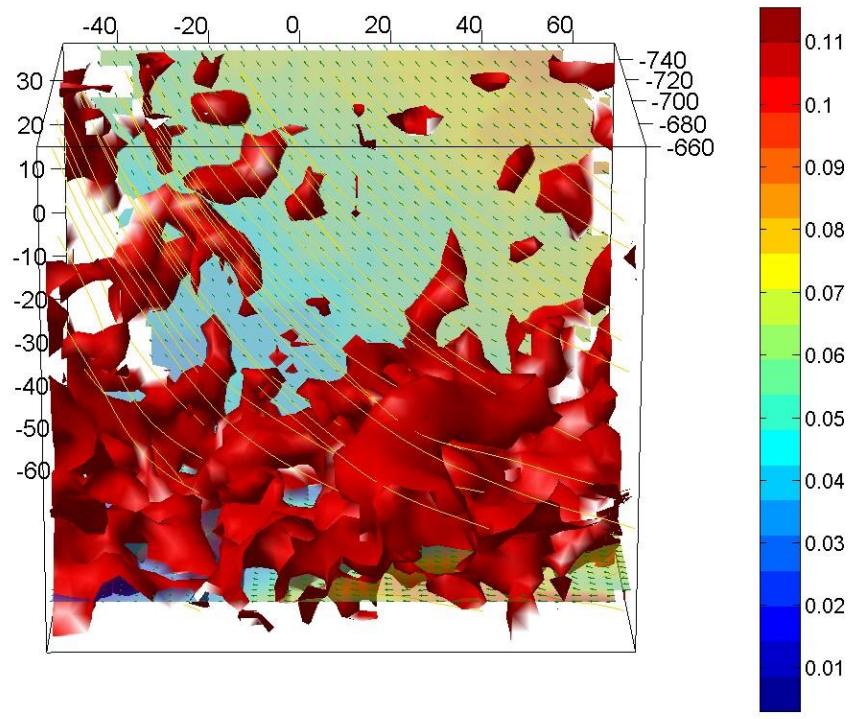

Figure 11. Near bed turbulence just before the flow reversal, Frame 13.

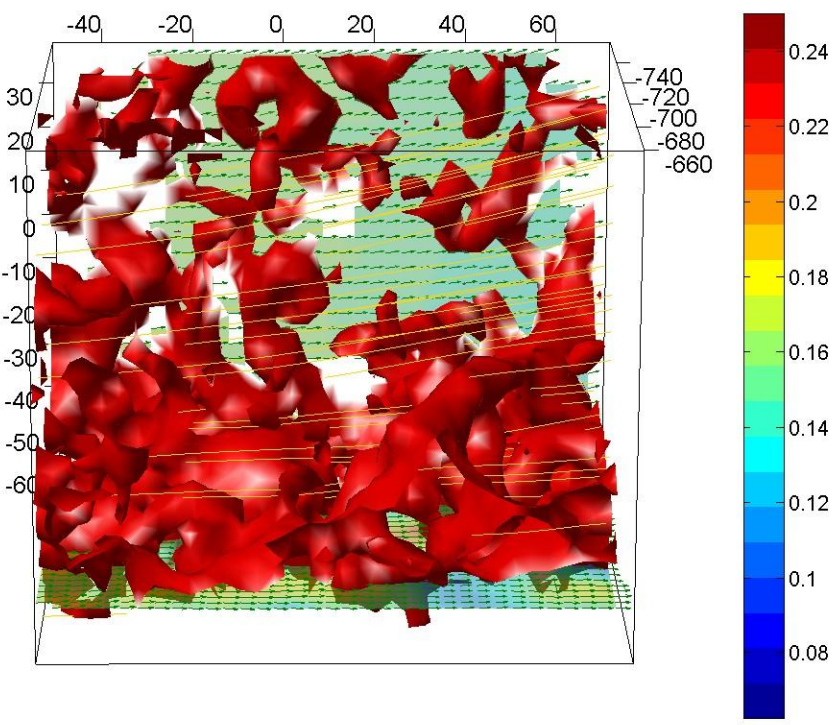

Figure 12. Ejection of vortices into the flow, Frame 15. 


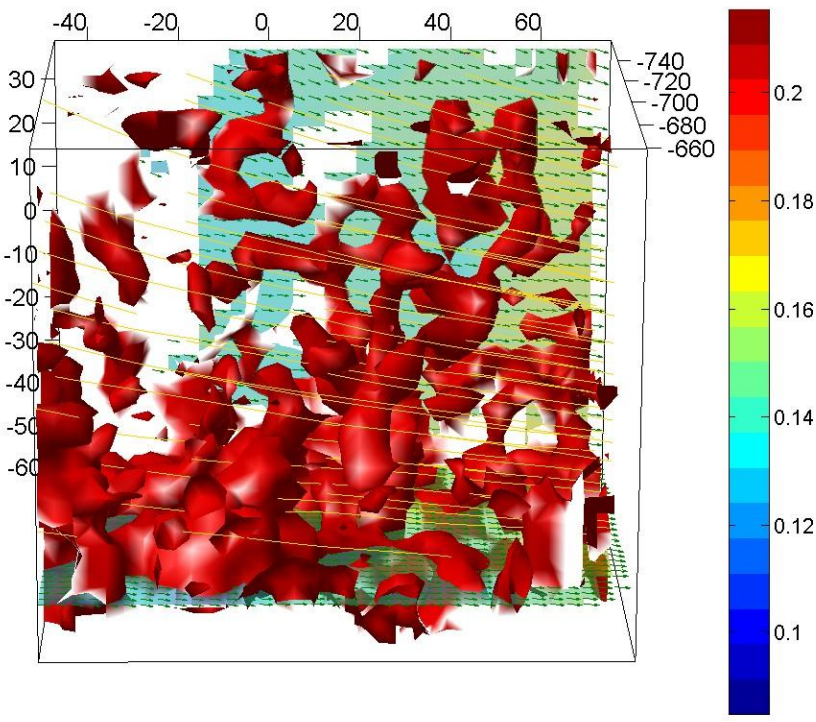

Figure 13. Ejection of vortices into the flow, Frame 19.

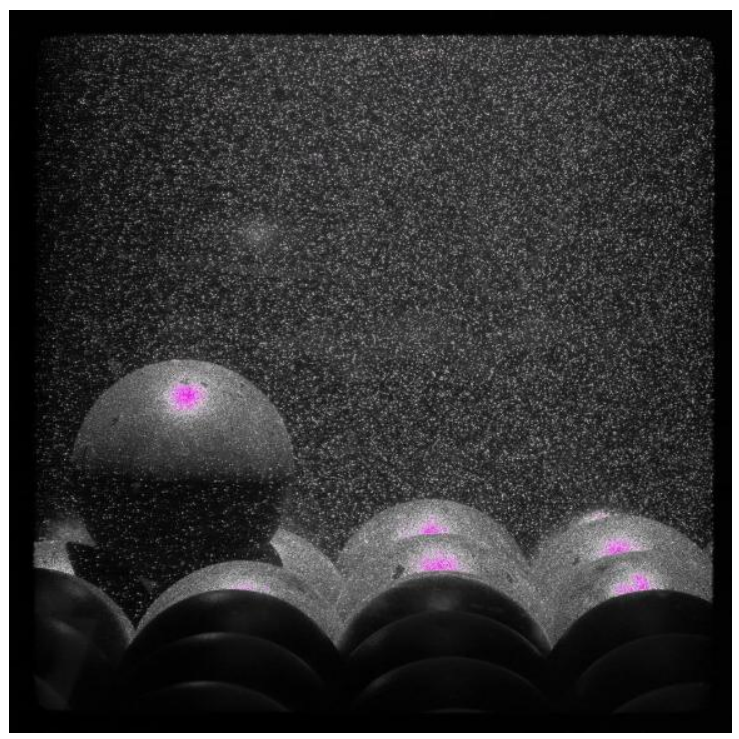

Figure 14. Raw V3V image of the $50 \mathrm{~mm}$ fully exposed sphere. 


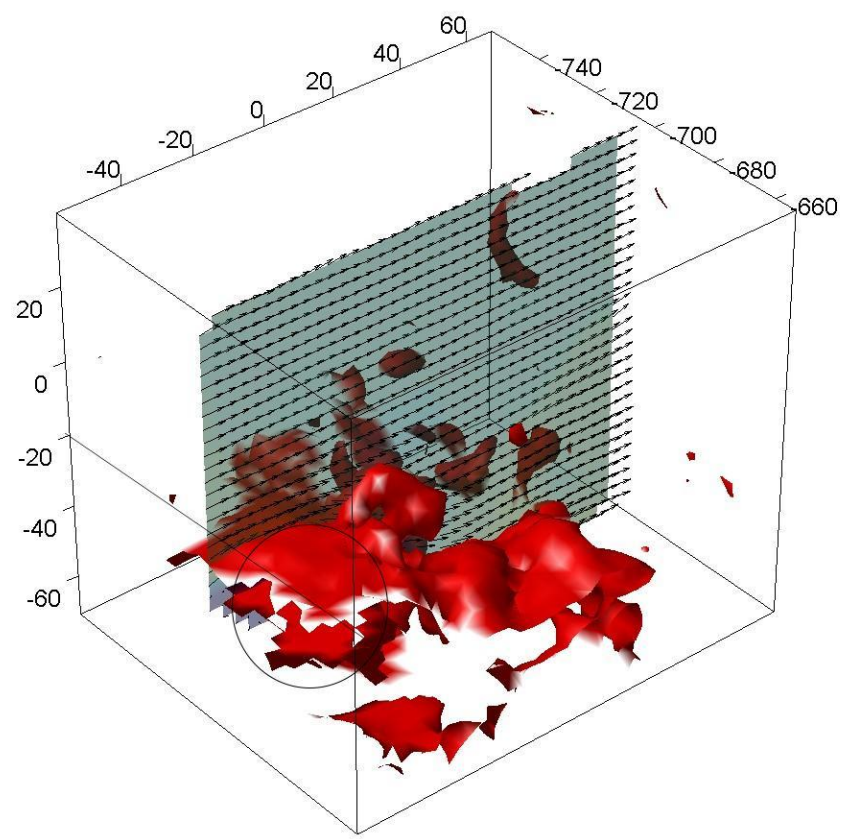

Figure 15. Vorticity isosurface around $50 \mathrm{~mm}$ sphere, Frame 62.

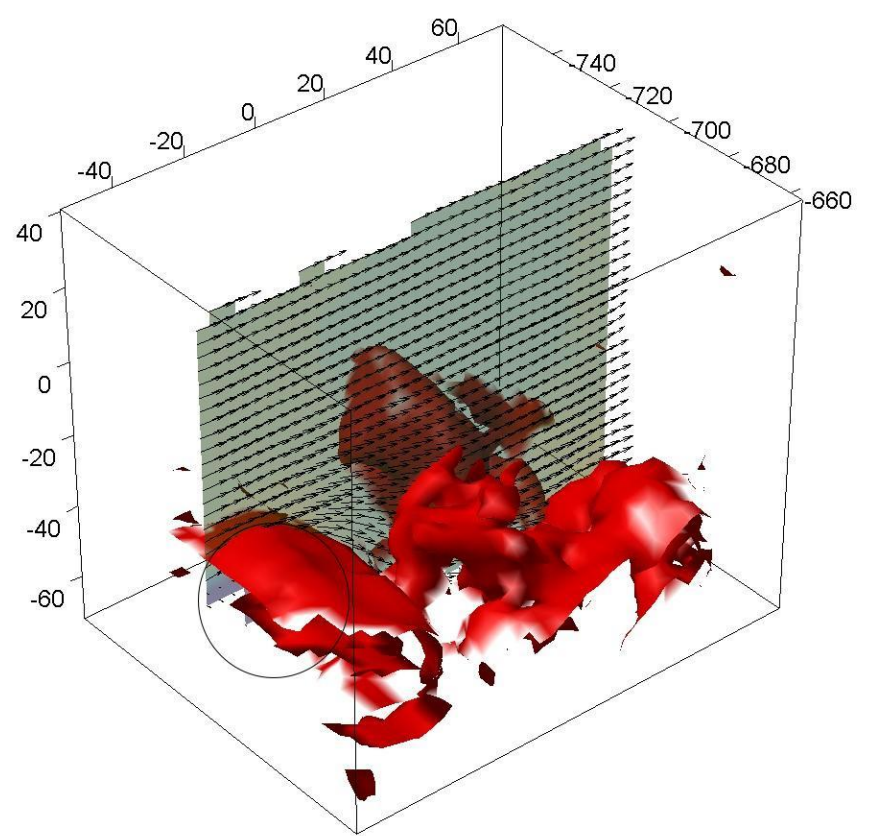

Figure 16. Vorticity isosurface around 50mm sphere, Frame 63. 


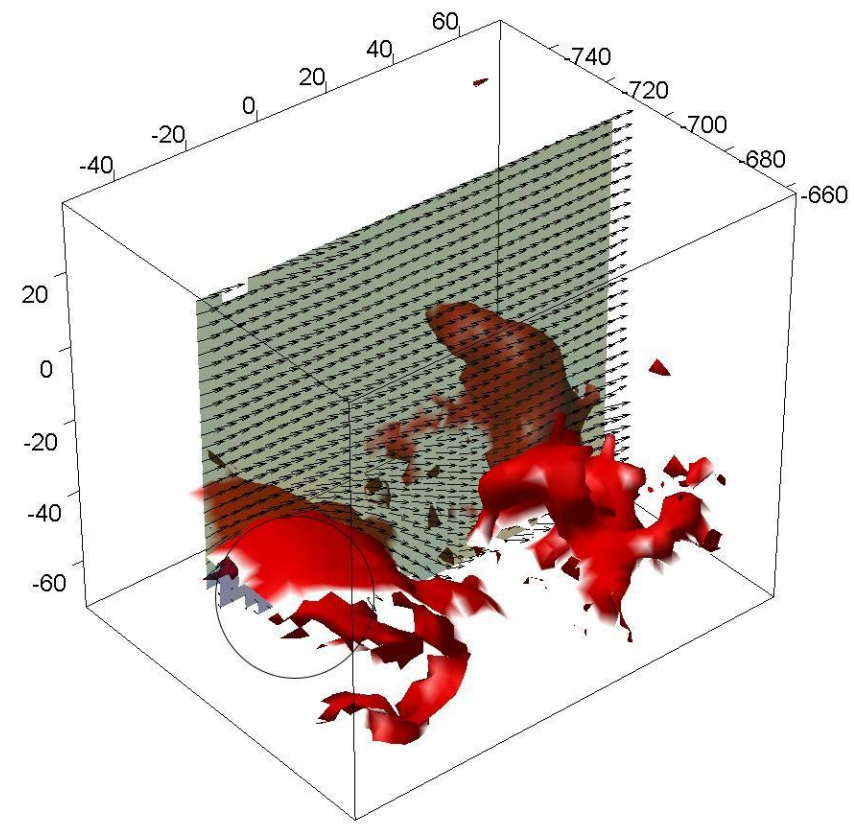

Figure 17. Vorticity isosurface around 50mm sphere, Frame 64.

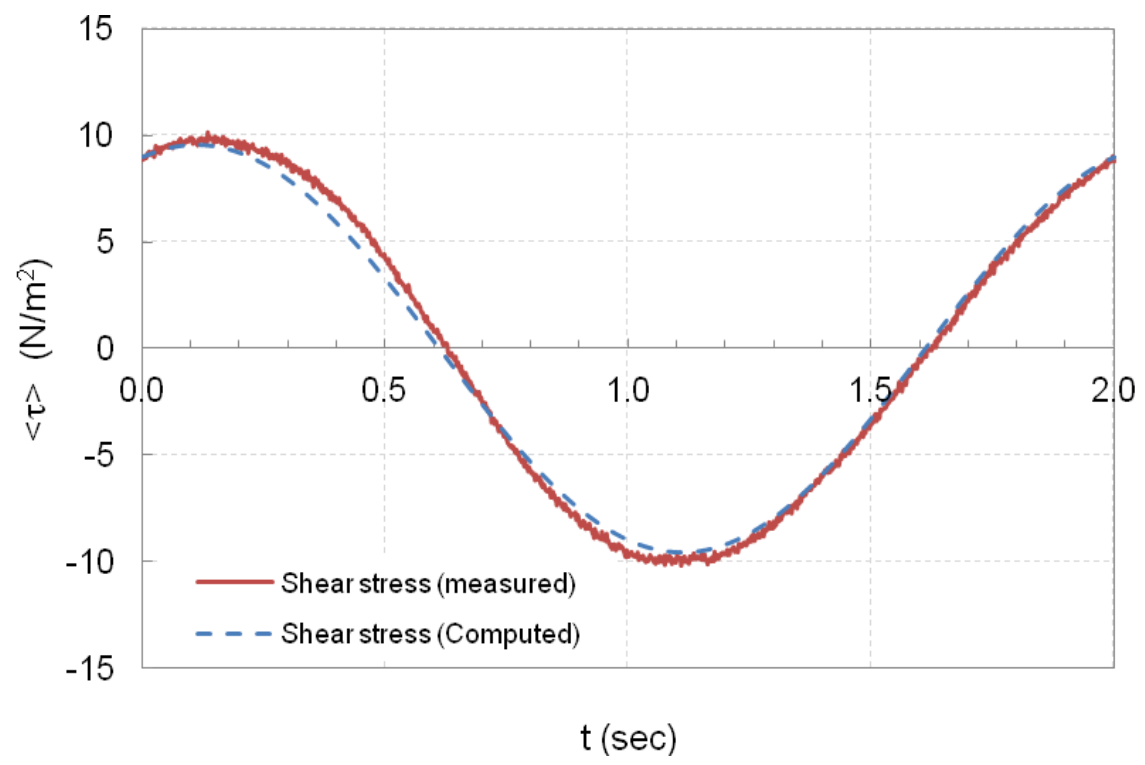

Figure 18. Shear stress computed using $<\tau>=0.5 f_{w} \rho U_{m}{ }^{2} \cos \left(\omega_{t}\right)$ superimposed on measured shear stress profile, $<\tau>$. $H=8 \mathrm{~cm}, T=2.0$ sec., $h=40 \mathrm{~cm}$. 


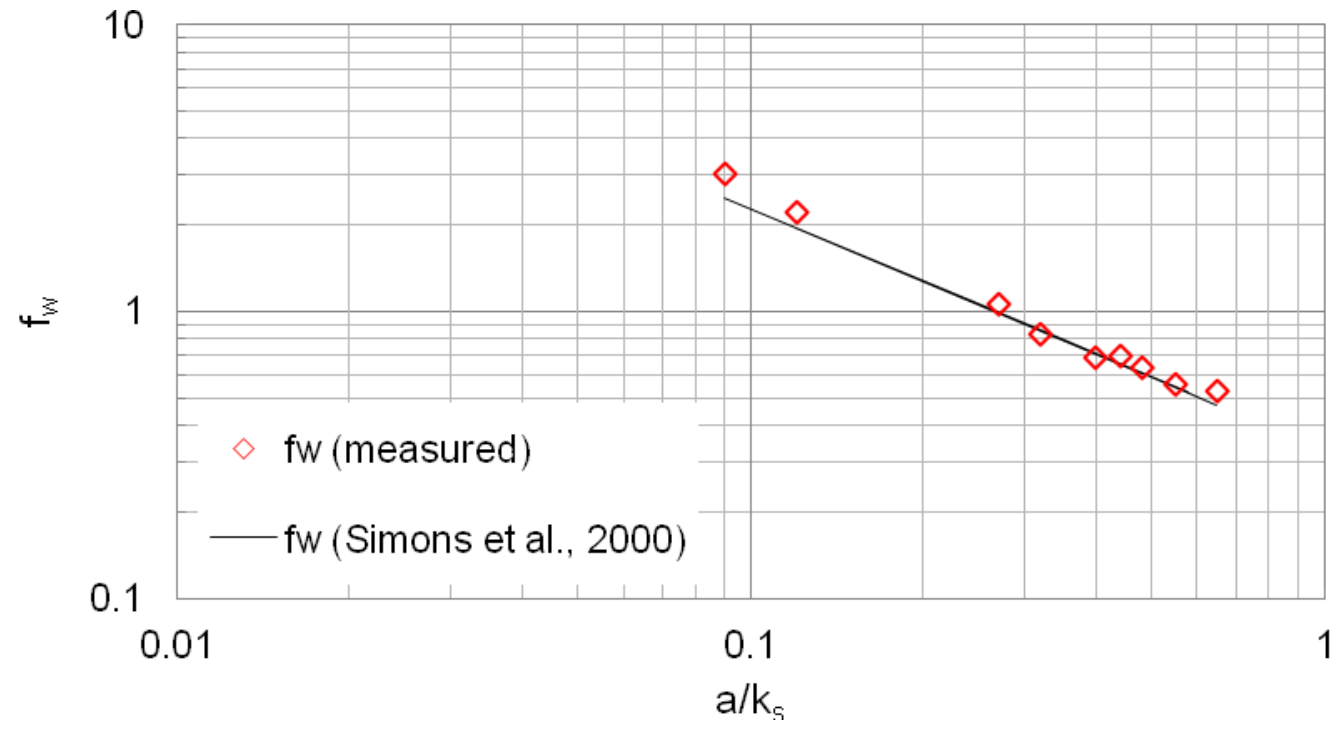

Figure 19. Measured friction factor compared with the relation $f_{w}=0.33\left(a / k_{s}{ }^{-0.84}\right.$ of Simons et al. (2000).

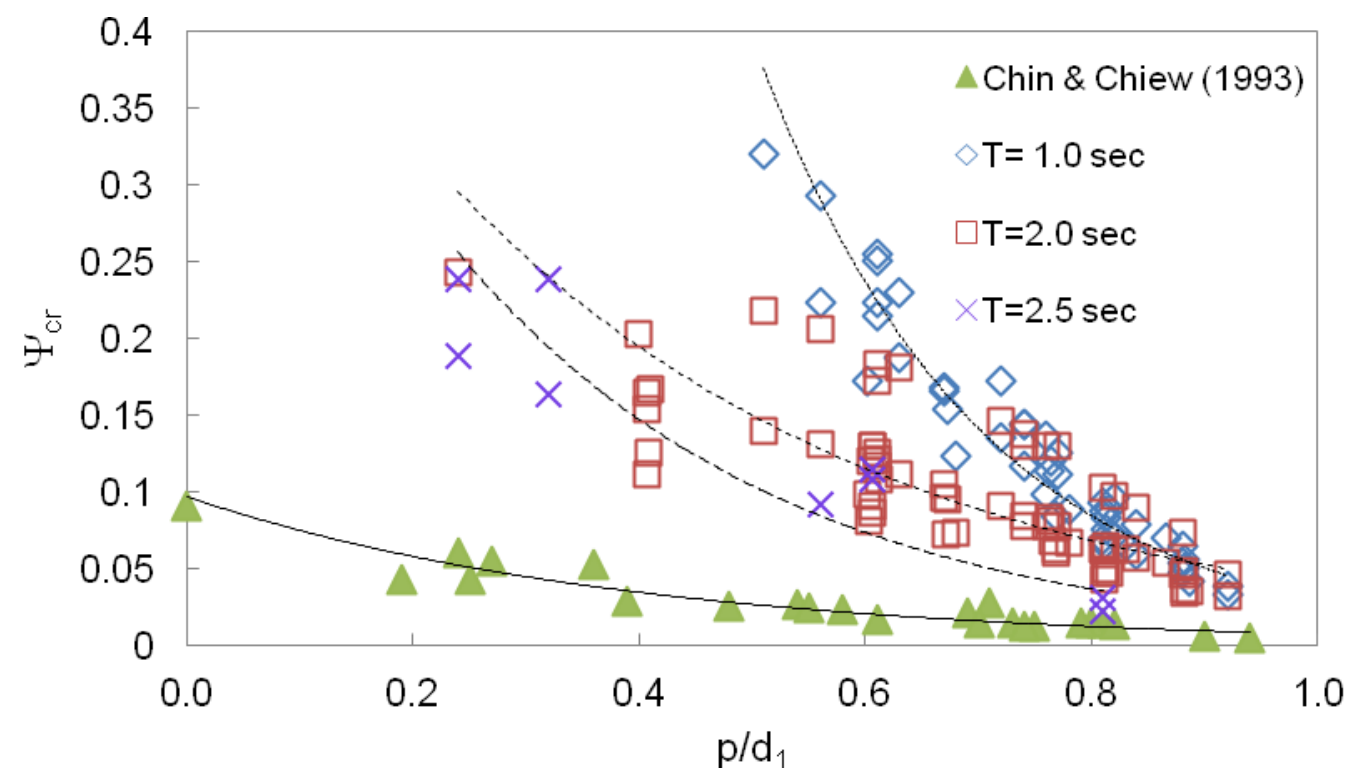

Figure 20. Measured critical shear stress data for different wave periods compared with data of Chin \& Chiew (1993) for uni-directional currents. 


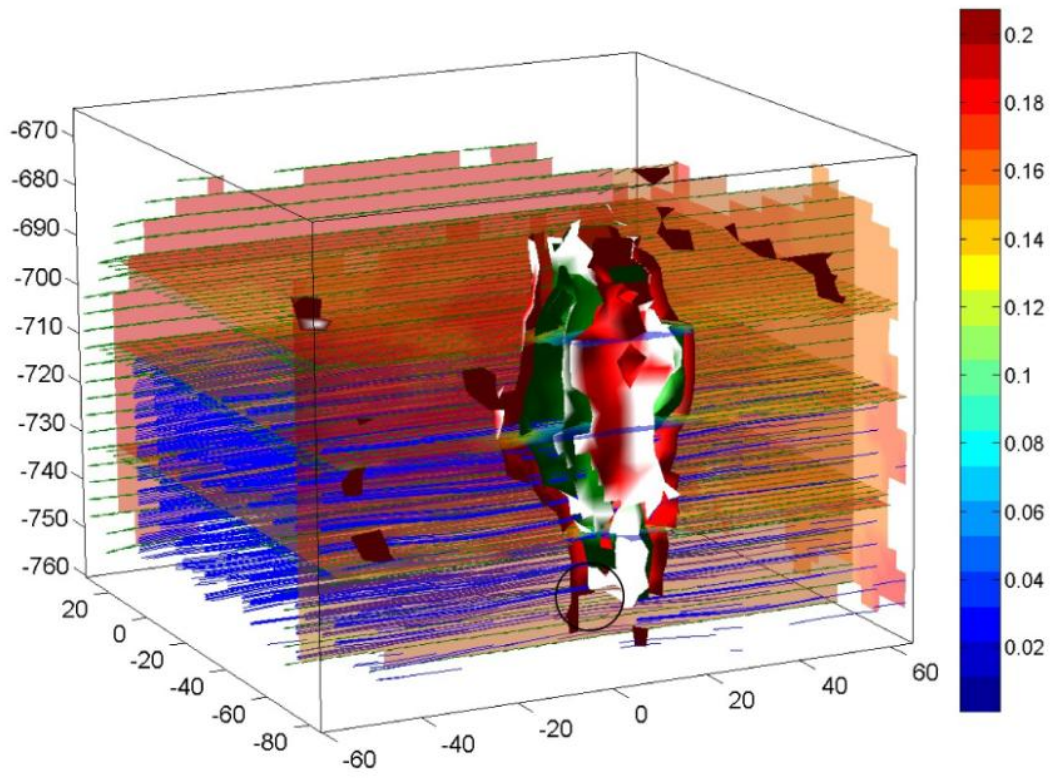

Figure 21. Residual vorticity structure on top of the $19 \mathrm{~mm}$ sphere at the peak velocity. $\mathrm{H}=9.5 \mathrm{~cm}, \mathrm{~T}=2 \mathrm{sec}$, $R e=2.1 \times 10^{4}, R_{d}=3518$.

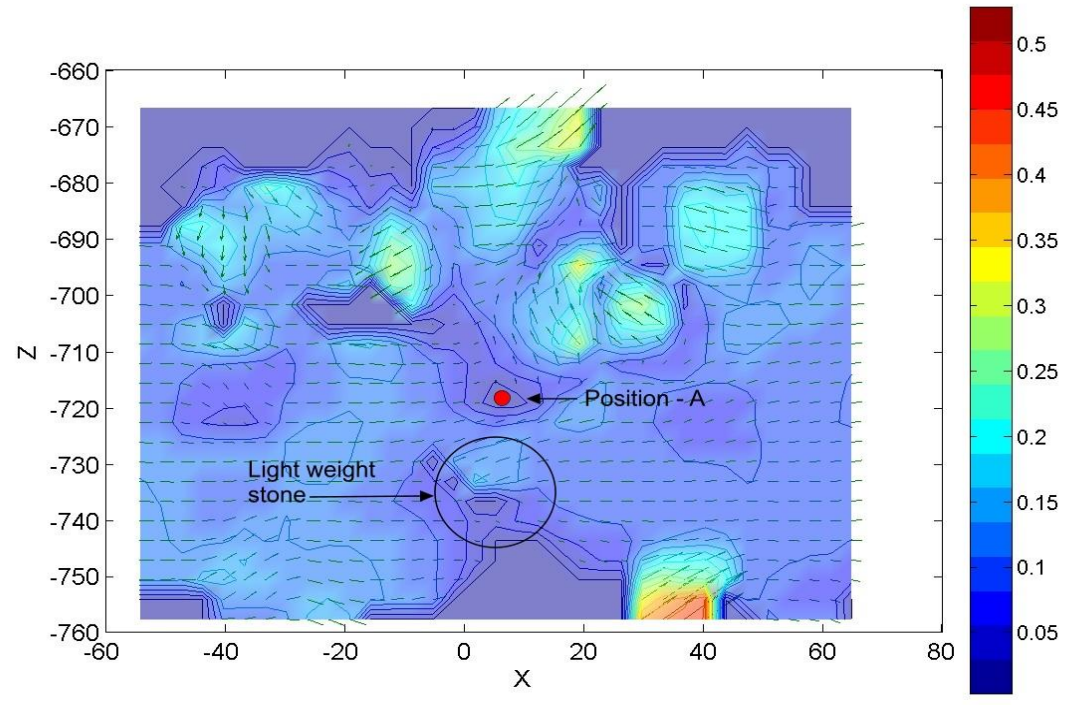

Figure 22. Position of the vorticity structure just before movement. $t=15.725 \sec$ (Frame 115) 


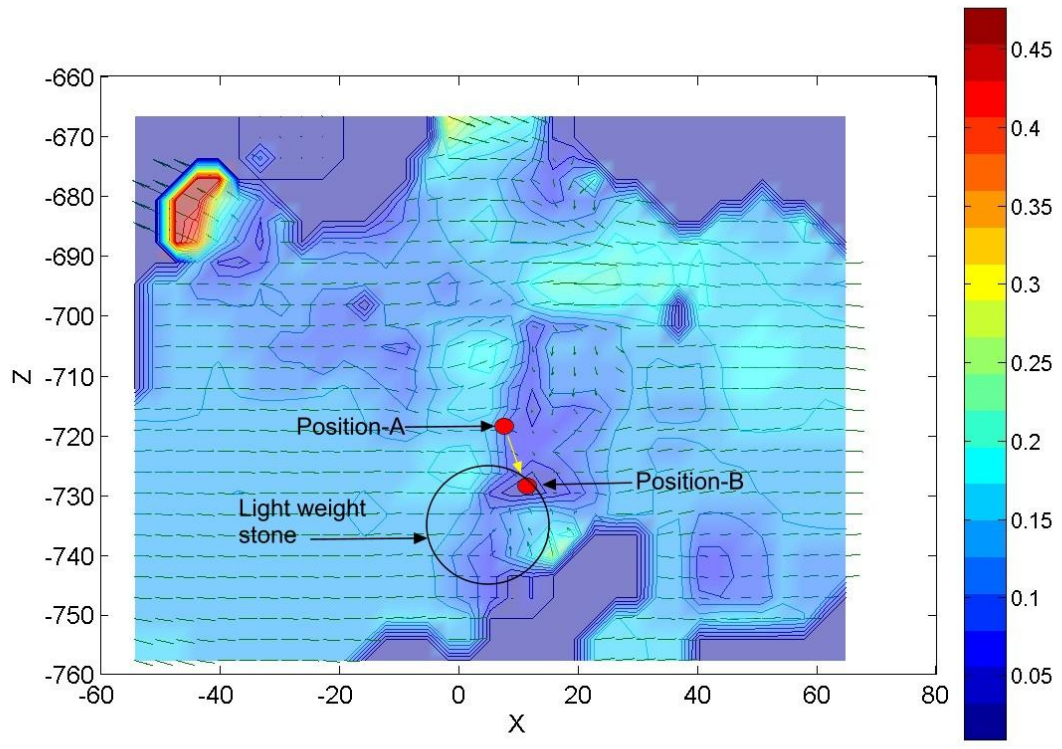

Figure 23. Position of the vorticity structure at stone displacement, $t=15.863 \mathrm{sec}$ (Frame 116)

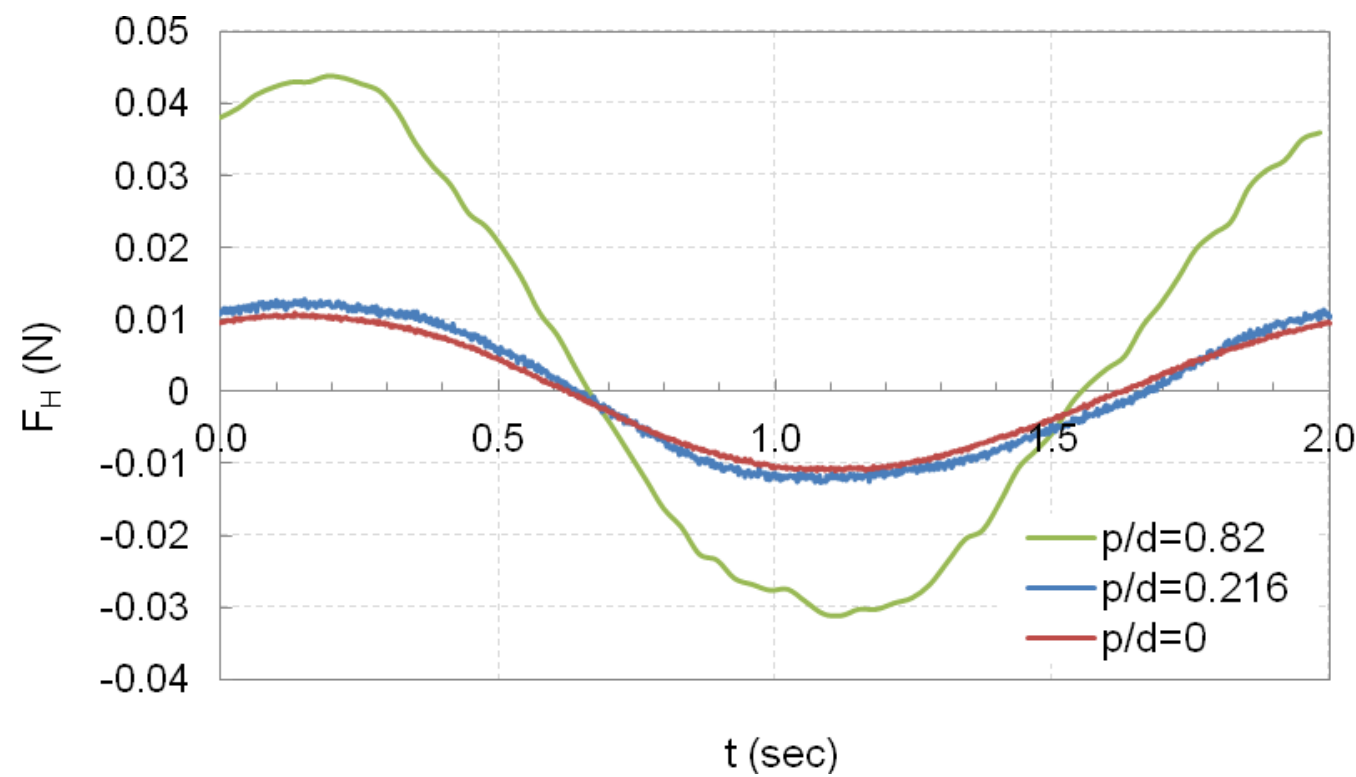

Figure 24. Measured horizontal force on a $50 \mathrm{~mm}$ spherical stone at different relative protrusion, $\mathrm{p} / \mathrm{d}$. 


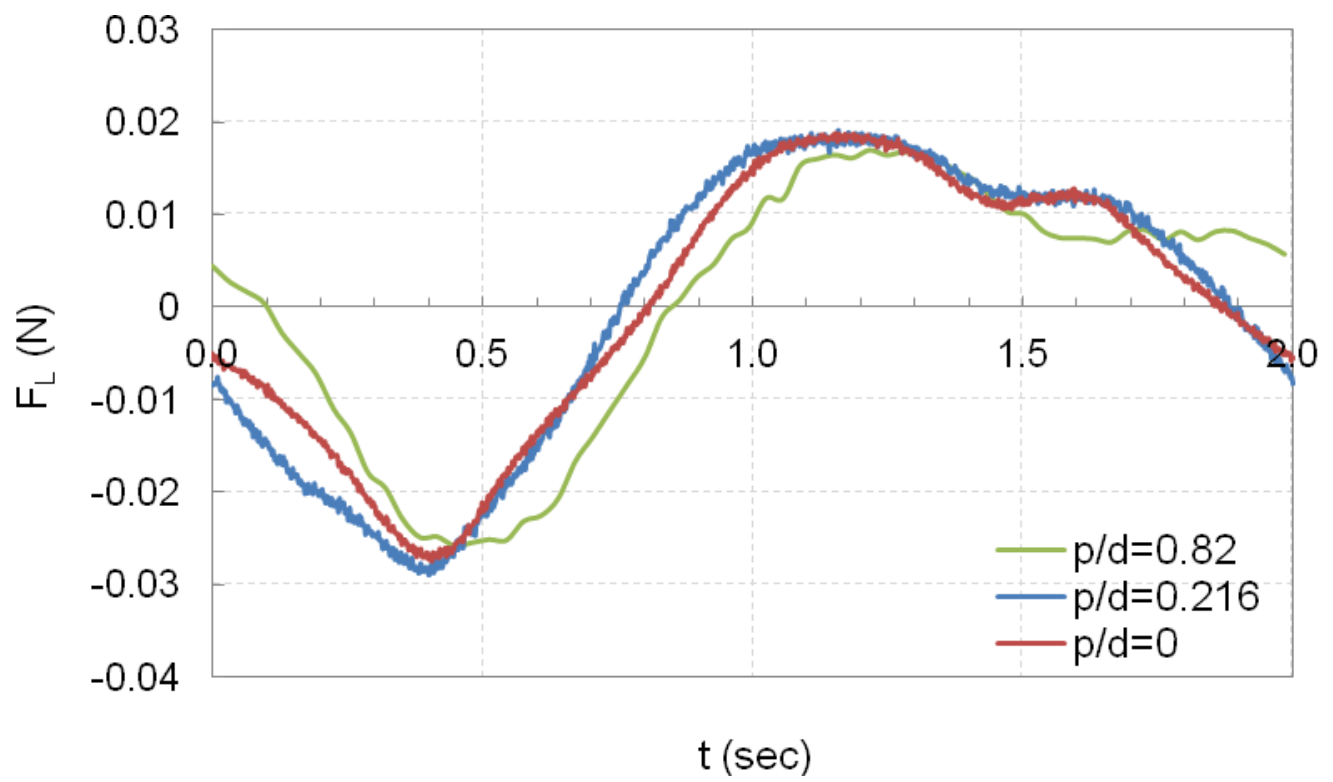

Figure 25. Measured uplift force on a $50 \mathrm{~mm}$ spherical stone at different relative protrusion, $\mathrm{p} / \mathrm{d}$.

\section{CONCLUSIONS}

Based on observations made in the tests the following conclusions can be made:

- The Shields critical shear stress has an exponential relationship with the stone protrusion. V3V measurements revealed that the remarkable stability observed for stones under waves in the present tests compared to currents is due to the fact that the flow was not fully developed at the moment of inception of motion. Therefore, the threshold critical shear stress observed for currents are applicable for waves under the field conditions where fully developed flow conditions prevail.

- It is confirmed by empirical experiments on spherical elements of varying density and size that stones in a bed protection are stable at $20 \%$ exposure.

- An "idealised" pressure gauge system suggests that the hydraulic datum is located $0.35 \mathrm{~d}$ below the crests of the roughness elements. This agrees with shear plate and other previously published data for friction factors.

- $\quad \mathrm{V} 3 \mathrm{Vmeasurements} \mathrm{suggest} \mathrm{that} \mathrm{the} \mathrm{uplift} \mathrm{induced} \mathrm{by} \mathrm{the} \mathrm{presence} \mathrm{of} \mathrm{vorticity} \mathrm{structures} \mathrm{above} \mathrm{a}$ stone is needed to displace a previously stable element.

\section{ACKNOWLEDGMENTS}

The authors are grateful to Dr Dan Troolin and Dr. Carsten Kykal for their support.

\section{REFERENCES}

Chin, C.O., and Y.M.Chiew. 1993. Effect of bed surface structure on spherical particle stability. Journal of Waterway, Port, Coastal and Ocean Engineering, 113(3), 231-242.

Kajiura, K. 1968. A model of the bottom boundary layer in water waves. Bulletin Earthquake Res. Inst., 46, 75-123.

Sleath, J.F.A. 1987. Turbulent oscillatory flow over rough beds. Journal of Fluid Mechanics, 82, 360409. 